D'une catastrophe à l'autre dans la Moskitia : de l'après-guerre à l'après-Mitch sur les ríos Wangki et Coco (Nicaragua)

\title{
Gilles Bataillon
}

\section{(2) OpenEdition \\ Journals}

Édition électronique

URL : http://journals.openedition.org/jsa/1417

DOI : $10.4000 /$ jsa. 1417

ISSN : 1957-7842

Éditeur

Société des américanistes

\section{Édition imprimée}

Date de publication : 1 janvier 2002

Pagination : 260-278

ISSN : 0037-9174

Référence électronique

Gilles Bataillon, « D'une catastrophe à l'autre dans la Moskitia : de l'après-guerre à l'après-Mitch sur les ríos Wangki et Coco (Nicaragua)», Journal de la Société des américanistes [En ligne], 88 | 2002, mis en ligne le 24 novembre 2005, consulté le 15 septembre 2020. URL : http://journals.openedition.org/jsa/ 1417 
—, 2002e. — « Convenção 169 da OIT é finalmente ratificada pelo Brasil », Últimas Notícias, 19/06/2002, ISA, Brasília.

Kopenawa, D., 2001. - "The rivers know our blood», in : B. Albert (ed.), Research and ethics : the Yanomami case (Brazilian Contributions to the Darkness in El Dorado controverse), pp. 115-121, CCPY-Pró-Yanomami (Documentos Yanomami, 2), Brasília.

Lobo, M. S. de C., K. M. P. Rodrigues, D. M. de Carvalho \& F. S. V. Martins, 2001. - " Report of the medical team of the Federal University of Rio de Janeiro on accusations contained in Patrick Tierney's Darkness in El Dorado ", in : B. Albert (ed.), Research and ethics : the Yanomami case (Brazilian Contributions to the Darkness in El Dorado controverse), pp. 15-42, CCPY-Pró-Yanomami (Documentos Yanomami, 2), Brasília.

Ramos, A. R., 2001. - " Commentary for the "Forum on Anthropology in public : perspectives on Tierney's Darkness in El Dorado" ", Current Anthropology, vol. 42, n 2, pp. 274-276. Republié en 2001 in : B. Albert (ed.), Research and ethics: the Yanomami case (Brazilian Contributions to the Darkness in El Dorado controverse), pp. 7-13, CCPY-Pró-Yanomami (Documentos Yanomami, 2), Brasília.

SANtos, R. V., 2002. — « Os Yanomami e a polêmica dos antropólogos », O Globo, 22/06/2002, Rio de Janeiro.

Santos, R. V. \& C. E. A. Coimbra Jr., 1996. - «Sangue, bioética e populações indígenas », Parabólicas, $\mathrm{n}^{\circ}$ 20, p. 7, São Paulo.

WEISS, K. et al., 1994. - « Optimizing utilization of DNA from rare or archival anthropological samples ", Human Biology, vol. 66, nº 5, pp. 796-804.

\section{2. - NICARAGUA}

\section{D'UNE CATASTROPHE À L'AUTRE DANS LA MOSKITIA : DE L'APRĖS-GUERRE À L'APRÈS-MITCH SUR LES RÍOS WANGKI ET COCO ${ }^{1}$}

Trois ans et demi après le passage de l'ouragan Mitch (novembre 1998), les villages situés le long du Wangki, le fleuve frontalier entre le Honduras et le Nicaragua, et de ses affluents - le Bocay et le Waspuc - présentent une variété de situations riches d'enseignements sur les avatars de l'aide au développement et de l'aide d'urgence. La vue que l'on en a depuis les bateaux qui assurent le transport des gens et des marchandises dans la région, et les commentaires de leurs pilotes et des autres passagers permettent de se faire une première idée de la situation.

La descente du fleuve de Wiwili à Waspam révèle un paysage tout en contrastes. Côté hondurien, dans le département d'Olancho, la forêt tropicale qui recouvrait les flancs des collines a généralement été essartée et transformée en prairie par des éleveurs métis venus des départements centraux du pays. Les villages sont quasi inexistants. On distingue en revanche des groupes de quelques maisons et d'étables appartenant à un chef de famille et à ses proches venus coloniser la zone. À ces maisons de planches et de tôles s'ajoute souvent une boutique où l'on vend de la 
nourriture aux gens de passage, de l'alcool, de la bière et des sodas, quelques habits, des bottes en caoutchouc, des instruments aratoires, des médicaments et des bonbons. Côté nicaraguayen, cette partie du département de Jinotega n'a pas encore été trop envahie par les colons hispanophones qui, plus en amont, ont détruit la forêt pour planter des caféiers, des haricots et du maïs, ou engraisser du bétail. Ici la forêt domine les petites montagnes calcaires, même si elle a été essartée autour des zones habitées. Les villages de cases en bambous refendus, en mauvaises planches ou en toiles plastiques alternent avec des villages manifestement reconstruits, les uns avec des maisons en briques d'adobe et toits en tuiles, les autres en planches avec toits de tôles, de palmes ou de tuiles. Ces villages reconstruits sont souvent dotés d'écoles en parpaings et en bois, aux toits de tôles fraîchement peintes. D'autres, tels Hamak, San Andrés Bocay ou Raïti, possèdent de plus toute une série d'équipements collectifs : postes de santé, réfectoires pour les enfants scolarisés, école secondaire, salles de réunion, maisons des femmes et des commissions de paix. On aperçoit aussi des constructions qui sont autant de locaux d'associations locales, d'organisations non gouvernementales (ONG) ou d'institutions internationales telle, ici, l'Organisation des États américains (OEA), qui ont pris en charge des programmes de distribution d'aide d'urgence ou des opérations de développement. On distingue enfin de nombreux drapeaux sandinistes, noir et rouge, ainsi que les affiches rose et jaune du candidat à l'élection présidentielle, Daniel Ortega, et, bien moins nombreux, quelques drapeaux rouges du parti libéral.

Les quelque cinquante kilomètres de rapides qui séparent Raïti de Karizal sont des deux cotés du fleuve le domaine de la forêt tropicale. On distingue ça et là quelques cacaotiers, vestiges des plantations d'une famille de commerçants miskitu-honduriens abandonnées lors de la guerre entre sandinistes et contras miskitus (1982-1988) et jamais exploitées depuis. L'entrée dans les basses terres miskitus ne se traduit pas seulement par la disparition des reliefs calcaires aux multiples petites falaises. Sur la rive hondurienne, la forêt primaire a été pratiquement partout détruite. Et l'on voit apparaître quelques villages dans un paysage fait d'un mélange de prairies, de plantations de bananes et d'une savane plus ou moins dense. Plus haute, et donc en principe plus préservée des crues, la rive nicaraguayenne est paradoxalement beaucoup plus peuplée, mais beaucoup moins défrichée. Si les villages se succèdent tous les cinq ou dix kilomètres, ils sont souvent séparés par des portions de forêts et pas simplement par des grandes étendues de savane. Et, comme en amont, on retrouve les mêmes contrastes entre des villages fort bien reconstruits ou dont l'habitat a été relativement épargné par la crue (Asang, San Carlos, San Esquipulas, Namahka, Wiwinak, San Jerónimo et Waspam, chef-lieu du municipe), des villages toujours à moitié en ruines (Santa Isabel, Sang Sang et Kitaski), et des villages en pleine reconstruction (Sixairi, El Paraiso ou Waspukta), mais aussi bon nombre de communautés où les projets de reconstruction sont à moitié paralysés (Pilphilia, Krin Krin ou Santa Fé). À la différence des communautés du département de Jinotega, aucune des communautés du municipe de Waspam, qui fait partie de la Région autonome de l'Atlantique Nord (RAAN), n'a bénéficié de la construction d'infrastructures ou d'équipements collectifs comme ceux réalisés sous la direction de l'oeA à Raïti ou à San Andrés Bocay. On aperçoit en revanche, quand on aborde les communautés, différents panneaux signalant que telle ou telle ONG s'est chargée des opérations de reconstructions post-Mitch. 
L'arrivée à Waspam donne une vue un peu caricaturale du Projet Waspam financé par la Union européenne (UE). On distingue en effet au premier chef la splendide maison particulière du responsable de projet ; demeure d'un luxe raffiné auprès de laquelle les autres constructions du Projet — bâtiments administratifs et ateliers — font bien pâle figure. La visite par la route des villages situés en amont de Waspam - Saupuca, Bilwaskarma et Kum - permet de découvrir une série de ponts reconstruits par l'uE en dépit du bon sens. Faute d'avoir employé suffisamment de ciment et de fers à béton, ces ouvrages sont déjà à moitié détruits ou impraticables quelques mois à peine après leur inauguration! On distingue aussi toute une série de latrines en zinc flambant neuves, offertes par la Croix rouge nord-américaine.

Dernière différence : la RAAN connaît une offre politique beaucoup plus diversifiée que le Jinotega. Depuis Raïti, qui marque la limite entre les deux zones administratives, les drapeaux de yatama (l'organisation indianiste de l'ancien dirigeant de la guérilla Brooklyn Rivera) se mêlent à ceux du parti régionaliste - le PAMUC comme à ceux des libéraux et des sandinistes.

\section{L'expérience de la guerre et des déplacements forcés}

Par-delà la variété de leurs situations, tous ces villages miskitus et mayangnas ont connu une même histoire de guerres et de destructions. Marquant la frontière entre le Honduras et le Nicaragua, le fleuve a été cinq ans durant (1982-1987) une sorte de ligne de front entre les guérilleros miskitus de la Contra et les forces armées sandinistes. C'est à Raïti et à San Carlos qu'eurent lieu en décembre 1981 les premiers affrontements opposant les combattants miskitus et les troupes de l'Ejército Popular Sandinista (EPS). C'est à Leimus, un peu en amont de Waspam, que les sandinistes se vengèrent des premières pertes qui leur furent infligées à Raïti et à San Carlos en assassinant une trentaine de mineurs miskitus et en violant leurs compagnes. Début 1982, les habitants de ces villages furent déplacés de force et installés dans les fincas caféières du département de Jinotega, ou dans des camps de regroupement tels ceux de Tasba Pry (8 000 habitants). Si la population des habitants des communautés situées en amont de Raïti n'eut d'autre choix que de se soumettre à cette évacuation forcée, une bonne moitié des 25 à 30000 habitants des communautés situées en aval choisirent pour une part de fuir au Honduras. Parmi ces derniers, 15000 furent pris en charge par le Haut-commissariat aux réfugiés (HCR) à Mocoron et 2000 firent le choix de s'installer sur la rive hondurienne du Wangki. Ces déplacements qui, pour l'ensemble des Miskitus et des Mayangnas, furent vécus comme une sorte d'exode biblique, furent d'autant plus traumatisants que beaucoup de gens assistèrent à l'incendie de leurs villages, de leurs champs et de leurs vergers, ainsi qu'à l'abattage de leur bétail par les membres de l'EPS, venus ofliciellement les protéger des attaques de la Contra. C'est dire qu'en quelques jours tous les habitants du Río Coco perdirent non seulement l'essentiel de leurs biens - instruments aratoires et vaisselle, vêtements, radios, machines à coudre et économies - leurs bêtes et leurs récoltes, mais qu'ils furent de surcroît privés presque tous des ressources de leurs terroirs respectifs. Ces incendies détruisirent aussi toute une série d'équipements collectifs : écoles, temples moraves et leurs registres d'État civil, postes de santé... Par-delà les destructions matérielles, c'est toute une organisation socio-politique et religieuse qui fut jetée à bas. Plusieurs pasteurs et 


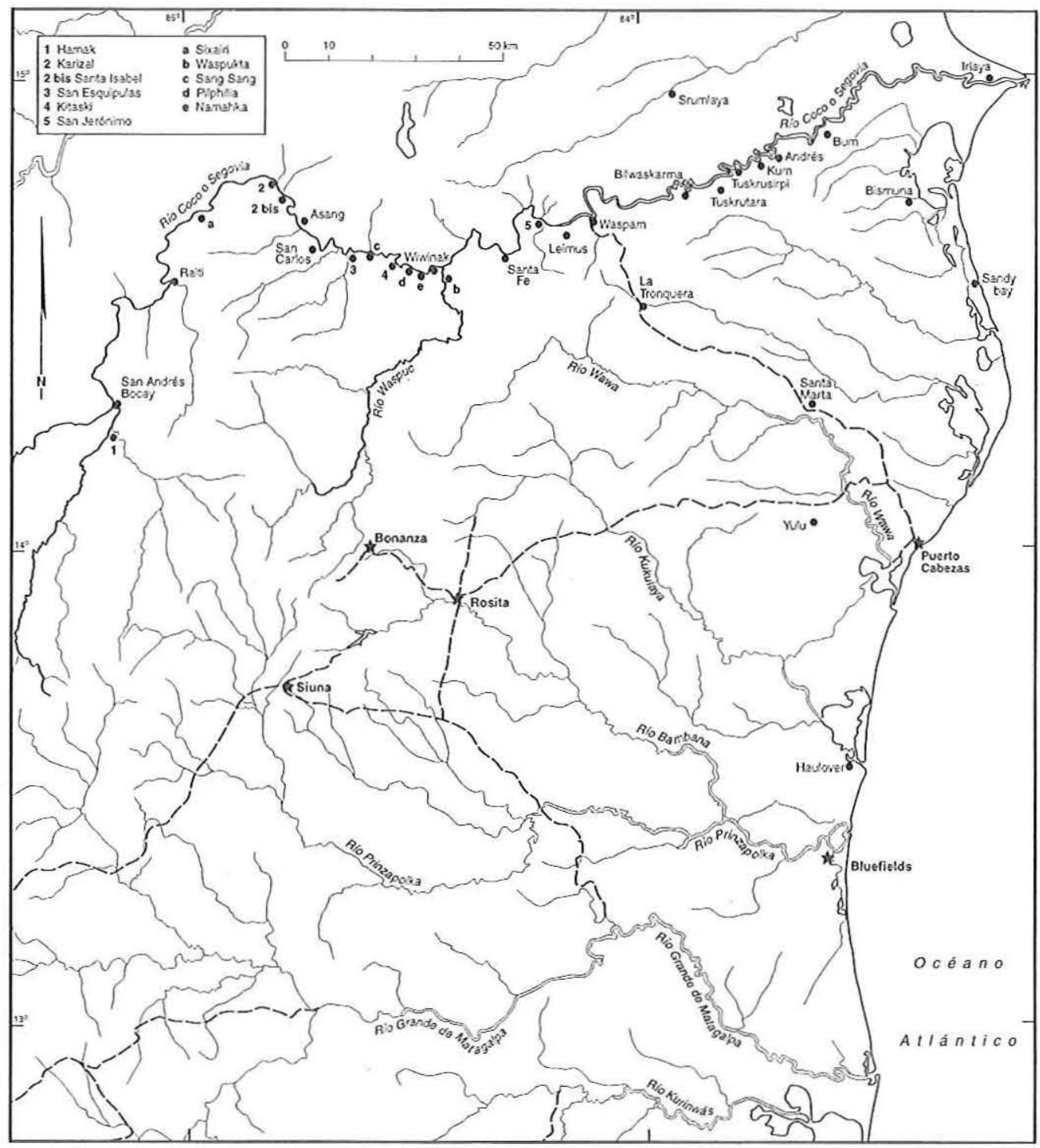

Fig. 1. - Région du Río Coco.

de multiples activistes du mouvement indianiste mISURASATA durent prendre la fuite pour éviter d'être emprisonnés. Tout l'appareil religieux de l’Église morave fut ébranlé, ses services sociaux et son système de formation, notamment l'hôpital et le séminaire de Bilwaskarma, furent fermés et transformés en bases militaires. D'une certaine façon, ces destructions signifièrent la fin des multiples améliorations de leur sort auxquelles étaient parvenues les communautés dans le cours des années 1960 et 1970. Des années d'expériences cumulées et de progrès dans les domaines de l'agriculture, de l'éducation scolaire, de l'habitat se virent anéanties en quelques jours. 
À ces premières épreuves s'ajoutèrent bientôt celles du transfert pour les personnes déplacées par l'armée et celle de la fuite au Honduras pour les autres, des marches dans l'ensemble passablement épuisantes tant physiquement que psychiquement. L'arrivée à Tasba Pry et dans les fincas pour les uns et la prise en charge par le HCR des autres ne signifièrent nullement la fin de leurs souffrances. Les premiers furent installés dans le plus total inconfort et la plus grande promiscuité sous d'immenses abris de toiles plastiques et soumis à la surveillance tatillonne des militaires, ou logés dans des conditions tout aussi désastreuses dans les hangars des fincas caféières. Les autres eurent plus de chance car traités avec plus de ménagement par le personnel du HCR. Tous firent néanmoins l'expérience d'attitudes et de comportements pour le moins discriminatoires à leur encontre. Les personnes installées dans les fincas et à Tasba Pry furent en butte au racisme et aux soupçons des autorités sandinistes qui voyaient en elles des "sauvages paresseux et ignorants » et des suppôts de ces "ennemis du Peuple » qu'étaient à leurs yeux les contras. Et tous furent considérés, dans un premier temps, comme potentiellement suspects et traités en conséquence. Si, par la suite, les habitants de Tasba Pry bénéficièrent d'une relative liberté de mouvement, ils n'en restèrent pas moins largement soumis à l'arbitraire des militaires. En revanche, les personnes déplacées vers le Jinotega ne connurent nulle amélioration durable de leur sort. Sans être stigmatisés d'une façon aussi catégorique, ni soumis aux procédés inquisitoriaux des responsables politico-militaires de l'EPS, les réfugiés au Honduras firent eux aussi l'expérience de situation de très grande précarité. Ils affrontèrent tout d'abord la méfiance et l'hostilité des responsables du HCR et des ONG travaillant à Mocoron qui, dans l'ensemble, étaient très largement favorables à la révolution sandiniste. Certains racontent ainsi comment une médecin française de Médecins sans frontières (MSF) put tranquillement leur déclarer : « Vous n'êtes que des paresseux et seul un gouvernement autoritaire [celui des sandinistes] peut améliorer le sort de gens comme vous ». Ils eurent aussi à souffrir, de 1982 à 1984, des exactions de la Contra miskitu comme de celles de l'armée hondurienne. Et ce n'est qu'à partir de 1985 qu'ils réussirent à instituer des relations plus égales tant avec la guérilla qu'avec le HCR. Enfin les 2000 réfugiés installés plus ou moins loin de leurs anciens villages sur la rive hondurienne du fleuve survécurent toujours dans les conditions les plus aléatoires, c'est-à-dire sans jamais recevoir aucune aide du HCR ou des ONG travaillant sous sa tutelle.

Ces années de guerre favorisèrent aussi une mobilité sociale tous azimuts et un profond renouvellement des élites locales. Les sandinistes surent attirer à eux de nombreux jeunes Miskitus en leur offrant la possibilité de poursuivre des études secondaires ou supérieures, et, ce faisant, de devenir instituteurs, professeurs de l'enseignement secondaire, travailleurs sociaux, techniciens ou mêmes ingénieurs. Ils attirèrent aussi des membres des anciens réseaux de pouvoir somozistes, notamment des maîtres d'école trop heureux de pouvoir faire oublier leur passé. Ils surent aussi promouvoir des femmes généralement réduites à des rôles subalternes dans le monde miskitu et mayangna. Enfin, la mise en place des Comités de défense sandiniste (CDS) fit le bonheur de nombreux intrigants souvent peu regardants sur leurs prérogatives. L'exil au Honduras fut pour beaucoup l'occasion d'acquérir de nouveaux statuts. Les promesses démagogiques et les pratiques dictatoriales de Steadman Fagoth, le principal dirigeant de la guérilla, fit naître chez certains le rêve d'accéder à des positions 
prééminentes et privilégiées. Apparut alors toute une camarilla de comandantes avides de se comporter en seigneurs de la guerre. Leurs exactions et les piétinements des opérations armées provoquèrent en retour tout un mouvement d'opposition démocratique au sein même de la guérilla. Et celui-ci, à l'emporter, promut toute une génération de nouveaux dirigeants beaucoup plus capables de travailler en synergie avec les ONG présentes dans les camps de réfugiés. De son côté, la désorganisation de l'Église morave facilita l'émergence des multiples groupes pentecôtistes qui eurent d'autant plus de succès que, forts du soutien des Églises américaines, ils furent à même d'offirir à certains jeunes hommes l'opportunité d'aller étudier au séminaire et de devenir pasteurs. Enfin l'aide " non létale » $[\mathrm{sic}]$ versée par le Département d'État à la Contra permit à beaucoup de guérilleros et à leurs proches de recevoir une formation professionnelle minimale : cordonniers, tailleurs, menuisiers, mécaniciens, comptables, dactylographes.

\section{Des retours chaotiques, des communautés politisées et divisées}

Loin de se dérouler de manière uniforme et ordonnée, les réinstallations des Miskitus et des Mayangnas dans leurs communautés d'origine furent le plus souvent autant d'opérations négociées au coup par coup, dans des conditions souvent très chaotiques. Les premiers à pouvoir revenir sur leurs terroirs d'origine furent les déplacés des hameaux stratégiques de Tasba Pry qui obtinrent fin 1985-début 1986 l'autorisation de voyager vers le fleuve pour préparer le retour. En revanche, les Miskitus et les Mayangnas du haut cours du Wangki et du Bocay, déplacés vers le Jinotega, puis à nouveau vers les llanos entre Rosita et Puerto Cabezas, ne parviendront à regagner leurs anciennes communautés qu'au début des années 1990. Parallèlement, si les communautés prises en charge par le HCR rentrèrent au Nicaragua en 1990 au lendemain de l'élection de Violeta Barrios de Chamorro, certains de leurs membres avaient commencé à se rapprocher de leurs communautés d'origine en s'installant en face de leurs anciens villages sur la rive hondurienne du fleuve. Par-delà les différences de calendriers, les conditions matérielles des retours des comunitarios diffèrent du tout au tout. Les habitants du Bocay et du haut Coco se réinstallèrent pratiquement sans aucune assistance hormis celle d'un pasteur allemand en contact avec l'Église morave. C'est dire qu'ils durent abandonner leurs maisons et la plupart de leurs biens et que c'est une fois sur place qu'ils reçurent l'aide d'organisations sandinistes qui appuyèrent leurs demandes de nourriture et de semis. De même la Commission interaméricaine de vérification (CIAv) de l'oEA dut-elle mettre sur pied tout un programme de déminage de la zone pour lequel elle n'avait au départ aucune ressource. La chance de ces déplacés fut de pouvoir se procurer des liquidités en vendant l'or qu'ils recueillaient dans les fleuves et les ruisseaux environnants. À l'instar de ces derniers, les quelque 2000 Miskitus qui avaient choisi de ne pas rallier Mocoron mais de s'établir plus bas sur le fleuve, entre Santa Isabel et le littoral, ne reçurent aucune aide pour se réinstaller ; ils eurent le plus souvent les plus grandes difficultés à obtenir que les organisations internationales comme le HCR prennent en compte leurs demandes. Les habitants de Tasba Pry ne bénéficièrent que d'une aide extrêmement limitée. Ils ne purent emporter que les tôles qui servaient de toiture à leurs maisons. Par ailleurs, du fait de leur départ, ils durent vendre dans de très mauvaises conditions leurs stocks de riz et de haricots. Les réfugiés sous la protection du HCR eurent droit à 
des aides nettement plus substantielles lors de leur rapatriement. Ils perçurent 200 lempiras (monnaie hondurienne ${ }^{2}$ ) par adulte et 150 par enfant, un jeu de vêtements neufs et des bottes en caoutchouc, des instruments de cuisine et de la vaisselle, des outils et des tôles, des semis et des vivres pour six mois. De leur côté, les guérilleros démobilisés et leurs familles reçurent peu ou prou une aide équivalente.

Toutes les communautés eurent non seulement à affronter la difliculté de remettre en état des villages détruits et des cultures abandonnées, mais souvent aussi le problème posé par la présence de mines posées là tant par la Contra que par l'EPS. Et il est encore aujourd'hui différentes zones autrefois agricoles ou forestières qui sont inutilisables du fait de la présence de mines qui explosent au passage d'animaux, d'enfants ou de chasseurs imprudents. S'ajoutèrent aussi à ces diffǐcultés matérielles des problèmes politiques, tout particulièrement dans les communautés dont la population avait été divisée en deux groupes : déplacés, parfois à leur demande vers les camps de regroupement sandinistes; exilés au Honduras, volontairement ou sous la pression de la guérilla. Dans le haut cours du fleuve frontalier, les retornados miskitus et mayangnas d'obédience sandiniste furent en butte à la suspicion et au racisme de colons ladinos liés pour beaucoup à la Contra. La situation fut d'ailleurs à ce point tendue que l'oEA dut créer des commissions de paix afin de réguler les conflits locaux. Dans le municipe de Waspam, les ex-réfugiés furent parfois persécutés par les membres de l'eps et de la police sandiniste. Enfin, dans de nombreux villages, les clivages entre ex-déplacés, ex-réfugiés et ex-guérilleros se perpétuèrent. Ainsi Asang resta longtemps divisée entre deux moitiés qui chacune avait ses autorités et son pasteur. La moitié sandiniste — « Moscou » - , ne reconnaissait que l'autorité de ses delegados, de son instituteur mis en place par les sandinistes, ainsi que de son pasteur. La moitié contra - "Washington » - ne jurait que par sa Junta élue sous la houlette du HCR, son instituteur ex-guérillero et son pasteur. Beaucoup d'autres villages, tel Wasla, connurent exactement les mêmes problèmes. Des problèmes qui ne furent d'ailleurs en rien purement et seulement "politiques", mais qui tinrent à des différences de ressources entre les ex-réfugiés et les ex-déplacés. Les premiers étaient jalousés par les seconds du fait de l'aide que leur avait consenti le HCR. Les seconds l'étaient par les premiers car, revenus depuis plus longtemps, ils avaient pu reconstruire leurs maisons en trouvant du bois plus facilement, remettre en état leurs plantations fruitières et leurs cultures. Les seconds s'étaient parfois emparés de parcelles qui étaient autrefois utilisées par les premiers ou leurs familles. Enfin, délégués, membres des juntes, instituteurs et pasteurs briguaient les mêmes postes et les avantages matériels qui leur étaient associés. Et ce n'est que petit à petit que furent trouvées des solutions à ces tensions, généralement sur la base d'arrangements ad hoc négociés entre chefs de réseaux familiaux. Beaucoup de ces villages furent en outre confrontés aux problèmes posés par la perte de toute une partie de leurs meilleures terres agricoles. En effet, si jusqu'au début des années 1980, les agriculteurs miskitus avaient pu utiliser des terrains situés sur la rive hondurienne du fleuve, ce fut désormais chose impossible : en effet, différentes familles métisses (miskitu-nicaraguayennes) avaient opté durant la guerre pour la nationalité hondurienne et s'étaient emparé entre temps de ces terres pour y pratiquer un élevage bovin extensif.

Il convient de prendre acte que la fin de la guerre civile, la défaite électorale des sandinistes devant la coalition de l'Union nationale d'opposition (UNO), le retour des 
comunitarios sur leurs anciens territoires ne signifièrent nullement un retour au statu quo ante. La plupart des habitants des villages en reconstruction revinrent très profondément désillusionnés par le cours des événements. Beaucoup avaient cru aux promesses des «lendemains qui chantent» faites par les sandinistes comme par certains contras. Nombre d'entre eux, pour ne pas dire la plupart, avaient perdu l'habitude de travailler pour subvenir à leurs besoins. Grâce au livret de rationnement, les habitants des asentamientos avaient en effet accès à toute une série de biens sans pour autant être obligés de travailler, et il en fut d'une certaine façon de même dans les camps du HCR. De plus, au Nicaragua, certains, bien en cour avec les autorités sandinistes, étaient aussi devenus experts dans l'art d'obtenir des faveurs en échange de leur activité politique. Mieux, tous savaient qu'un petit nombre avaient su user de positions politiques pour, non seulement obtenir « légalement » un accès privilégié à certains biens, mais encore utiliser des postes de responsabilités pour détourner, à leur profit ou à celui de leurs dépendants, différents biens, et ce, en toute impunité, comme le démontrèrent les lois dites de la Piñata. Côté Contra, certains comandantes agirent de la même façon en s'appropriant quantité de dons venus des États-Unis, là aussi dans la plus totale impunité. D'autres, tels les négociateurs de la Paix de Yulu en 1985, monnayèrent en espèces sonnantes et trébuchantes leur ralliement à cette " paix des braves ». La vie dans les asentamientos, les camps de réfugiés ou la guérilla, rendit les plus jeunes complètement étrangers aux difficultés du métier d'agriculteur tout en les habituant à la présence de services sanitaires et d'embryons de services sociaux sur lesquels compter. En bref, un certain idéal de travail ascétique qui avait été promu et valorisé par le clergé morave pendant des décennies fut battu en brèche. S'imposèrent, en revanche, non seulement des habitudes de dépendance envers l'État ou les organismes internationaux, mais aussi des pratiques d'échange de loyautés politiques contre des faveurs personnelles. On s'habitua aussi à la possibilité de réaliser, dans la plus grande impunité, des détournements de fonds étatiques ou para-étatiques. Enfin, chez beaucoup prévalut l'idée que les souffrances qu'ils avaient endurées, du fait des déplacements forcés ou de leur participation à la guerre, devaient être reconnues et de ce fait donner lieu à indemnisation, sous une forme ou une autre.

\section{Les premiers projets de réhabilitation et d'aide au développement}

C'est donc dans des communautés pour le moins fragilisées que prirent place les premiers projets de réhabilitation. Et quelles que furent les difficultés que durent surmonter les promoteurs de ces initiatives, celles-ci furent assez généralement couronnées de succès. Conçus le plus souvent à la demande des comunitarios, présentés par ces derniers à des ONG et à des fondations installées à Waspam, le chef-lieu du principal municipe du Río Coco, ces projets furent chaque fois relativement modestes et donc facilement identifiables : reconstruction d'écoles, de postes de santé et de maisons communautaires; amélioration des puits ; construction de latrines. Quels qu'en furent les opérateurs, le Projet Wangki de l'ue, une ong locale comme PanaPana ou encore Médecins du Monde (MDM), le principe fut chaque fois peu ou prou le même. Les bénéficiaires du projet se devaient de fournir la main d'œuvre pour la construction, tandis que l'organisme subventionnant l'opération faisait don des matériaux de construction (zinc, clous, ciment, grillage, fil de fer et outillage) ou bien 
pourvoyait aux moyens de leur extraction (scies, haches, tronçonneuses et essence). Dans les cas de constructions un peu élaborées, les bailleurs de fonds payaient en outre les charpentiers-menuisiers et les maçons dont les savoir-faire étaient indispensables à la bonne marche des chantiers. Les autorités communautaires n'eurent généralement pas de peine à mobiliser la main d'œuvre nécessaire à ces constructions. Ces opérations étaient dans l'ensemble réalisées en temps et en heure ou avec des retards parfaitement acceptables. MDM forma aussi avec un certain succès des leaders de santé. De la même façon, une organisation comme Vétérinaires sans frontières (VSF) fut en mesure de monter un projet de formation à l'élevage bovin et ovin. Sa mission (1993-1996) forma des Grupos de interés agropecuario (GIA), un peu sur le modèle associatif des GAEC français, dans différentes communautés. Elle eut la sagesse, à la fois de distribuer des têtes de bétail aux membres de ces GIA, mais aussi de les impliquer doublement dans l'opération : en mobilisant leur force de travail et en les faisant participer symboliquement à l'achat des médicaments et des vaccins pour le bétail. Parallèlement, dès le début des années 1990, les responsables d'une ONG miskitu comme Pana-pana posait le diagnostic suivant : le développement local nécessitait certes des crédits et des appuis étrangers à la région, mais ceux-ci n'étaient en rien un dû ; il s'agissait d'une opportunité qui n'aurait qu'un temps. Ils entendaient aussi rappeler au travers le nom de leur association - pana-pana signifie " entraide » - qu'il existait une tradition d'aide mutuelle, de don associé à un contre-don et une éthique du travail qui avaient été au fondement de l'expérience sociale des Miskitus. Forts de ce constat, ils vont lancer avec un certain succès différents projets de microcrédit dans plusieurs communautés du municipe de Waspam, comme dans d'autres zones de la Moskitia. Enfin, conscientes du fait que, depuis la fondation du premier mouvement indianiste - alpromisu, 1974 - la question des titres de propriété des terres miskitus et mayangnas était un problème récurrent dans les rapports entre les communautés indiennes et les gouvernements centraux, deux ONG nicaraguayennes reprirent à leur compte le projet de misurasata (1980) consistant à dresser une carte des territoires indigènes. Appuyée par The Nature Conservancy (TNC), ALISTAR dressa les cartes des terroirs mayangnas et miskitus du Río Bocay, du haut cours et du cours moyen du Río Coco. Mieux, ses membres commencèrent à poser les bornes de ces terroirs. Et des membres du CIDCA, en association avec le Central American and Carribean Research Council (CACRC), firent peu ou prou le même travail plus en amont. Ici encore, ces opérations résultèrent à la fois d'une prise en compte de demandes émanant des communautés et de la participation de leurs membres, et d'un financement exogène. Un tel financement fut indispensable à la fois pour former certains des comunitarios à l'usage des relevés GPS et à l'achat de ce matériel, comme au paiement de dessinateurs capables de dresser cartes et plans, ou de rédacteurs capables de produire brochures et rapports. Reste que, quelle que soit la qualité de ce travail, la reconnaissance légale de ces terres indiennes est toujours bloquée par l'incapacité du parlement nicaraguayen à voter une loi sur ce sujet.

$\mathrm{Si}$, dans l'ensemble, les projets évoqués ci-dessus réalisèrent très honorablement les objectifs qu'ils s'étaient fixés, il en est d'autres, tout aussi nombreux et sans doute largement aussi importants par leur poids financier; qui donnèrent lieu à de considérables détournements de fonds et finirent en fiasco. Les cas les plus exemplaires furent ceux de fonds pour la construction d'écoles et d'églises, de distributions incontrôlées 
de semis, de matériel agricole, de moyens de transport (bateaux et moteurs hors-bord) et de médicaments. Des donateurs étrangers, laïques ou religieux, ong ou agences gouvernementales telles l'AID, répondirent ainsi de bonne foi aux demandes émanant de la municipalité de Waspam ou d'ONG locales, telle IDSIM, l'oNG de l'Église morave, ou de différentes oflicines sandinistes. Mais, loin de veiller à la bonne exécution des projets, les bailleurs de fonds n'envoyèrent jamais personne sur place pour juger de l'avancement des constructions ou des modalités de la distribution des biens ou des crédits. Ce manque de suivi ne permit pas seulement les premiers détournements, mais accrédita l'idée que ceux-ci étaient, dans une certaine mesure, acceptables. En effet, rares furent les organismes qui, à l'image de l'Agence canadienne pour le développement, suspendirent purement et simplement les programmes là où la concussion était patente, mais encore obligèrent ces nouveaux « chevaliers d'industrie » à rembourser les biens mal acquis sous peine de se voir poursuivre en justice. Ainsi la CIAV-OEA eut-elle certes la sagesse de fermer un programme de développement agricole et de construction de silos pour la commercialisation du riz que d'anciens démobilisés de la Contra pillaient systématiquement. Et si, échaudée par l'expérience, elle se refusa à participer dorénavant à de telles entreprises dans la région, elle ne fit pas pour autant le choix de dénoncer publiquement les faits et d'intenter une action en justice contre les prévaricateurs. De même, les responsables européens du Projet Wangki suspendirent avec raison leurs opérations lorsque Steadman Fagoth, devenu gouverneur de la Région autonome de l'Atlantique Nord, voulut imposer son neveu comme codirecteur du projet et, ce faisant, organiser sa mise en coupe réglée. Mais eux aussi se refusèrent à faire ofliciellement toute la lumière sur les raisons qui motivèrent leur départ.

Force est de constater que, dès les lendemains de la réinstallation des déplacés, apparaissent chez les ONG et les agences prenant en charge des projets humanitaires ou de développement deux types d'action. L'une de ces praxis s'attachera à définir des objectifs et des projets au contact des populations, impliquera les bénéficiaires en les faisant participer non seulement comme récipiendaires de l'aide, mais en les responsabilisant, à la manière de vsf ou de Pana-pana. À cela s'ajoutera la capacité d'effectuer un suivi minutieux des opérations, seul à même d'éviter des opérations de grivèlerie. Certains auront même le courage de rendre publics les désaccords et les points de friction entre les exécutants d'un projet, ses bénéficiaires et les bailleurs de fonds. À l'inverse, une autre praxis sera à la fois infiniment plus distante face aux demandes formulées par les populations locales et plus soucieuses d'exécuter mécaniquement des distributions massives de nourriture, de médicaments, d'instruments aratoires ou de semis. Cette distance ira de pair, chez certains responsables des institutions bailleuses de fonds, avec une remarquable méconnaissance de la Moskitia et de son histoire. Jamais ceux-ci ne prendront le temps d'enquêter sur l'histoire récente de la zone, sur les péripéties de la guerre ou sur les tentatives de développement des années 1960-1970. Cela s'accompagnera d'une incroyable tolérance face aux ratés sans nombre de ces opérations par trop pensées à distance des réalités locales. Les inventaires et les inspections visant à s'assurer du bon fonctionnement de ces opérations à grande échelle seront des plus superficiels, favorisant largement les détournements tous azimuts.

La première de ces façons de faire sera généralement l'apanage d'ong locales aux moyens relativement modestes, composés de déçus de l'action politique. Leurs mem- 
bres aspirent généralement à une professionnalisation dans l'humanitaire ou le développement et sont donc peu tentés d'utiliser ces ressources pour se constituer une clientèle politique. Cette façon de travailler sera aussi généralement associée à des projets d'une certaine technicité impliquant durablement les étrangers venus en mission sur le terrain comme ce fut le cas avec le projet de vSF, ou encore les premiers projets d'ALISTAR et du CIDCA. Du coup, ces projets parviendront aussi pour une part à briser les féodalités politiques sandinistes/contras héritées de la guerre. Pana-pana et le CIDCA feront travailler côte à côte d'ex-guérilleros et des proches du Front sandiniste de Libération nationale (FSLN). La seconde façon de travailler va d'emblée s'inscrire dans un certain nombre d'ambiguïtés qui furent celles des différentes oNG qui existaient dans la région avant la rapatriation des réfugiés. Pour la plupart liées à l'Église morave ou au FSLN, celles-ci étaient des organismes dont les dirigeants détournaient, au vu et au su de tous, les dons venus de l'étranger. Et beaucoup de donateurs tolérèrent ces pratiques arguant de la pauvreté ou d'une supposée "culture locale ». Aussi, quand différents projets de développement financés par l'UE, l'AID ou l'OEA furent mis en place dans la région, certains de leurs futurs bénéficiaires, ex-contras ou partisans du FSLN, virent-ils en eux l'opportunité d'accéder, eux aussi, à des postes particulièrement lucratifs. Mieux, beaucoup cherchèrent à utiliser ces projets à des fins politiques. Une première technique fut d'user des ressources mises à disposition par les ONG ou les projets de développement pour financer les campagnes électorales tant lors des élections régionales de 1994 que des élections générales de 1996. Une seconde technique consista à proposer l'échange des voix contre la promesse de projets de développement pour la communauté ou d'avantages en nature pour les agents électoraux. Ces dérives furent en outre favorisées par le souci de différents bailleurs de fonds de travailler coûte que coûte dans la zone, le plus souvent au nom d'objectifs strictement bureaucratiques - un budget déjà attribué, la peur panique du scandale public - et non au regard d'une connaissance préalable des problèmes. S'ajoutèrent aussi des motifs parfaitement prosaïques, au premier rang desquels la volonté de conserver des emplois souvent fort bien rétribués pour les responsables locaux desdits projets. Il faut enfin souligner que, si ces attitudes peuvent souvent être associées à certaines $\mathrm{ONG}$, comme à certaines agences internationales, beaucoup d'intervenants oscillèrent entre ces deux « options ». Ainsi, Pana-pana fut pendant un moment la proie de politiciens liés à Brooklyn Rivera et connut alors certaines malversations. La scission d'une partie de ses membres lassés de ces pratiques, qui constituèrent une nouvelle ONG, la FURCA, et les remontrances d'une partie des bailleurs de fonds permirent de redresser la situation. Après des débuts prometteurs, la fondation ALISTAR se transforma à l'inverse peu à peu en une ONG surtout soucieuse de faire du prosélytisme pour le FSLN et de moins en moins capable d'efficacité sur le terrain.

Certaines de ces dérives furent renforcées par deux autres facteurs, l'un inhérent aux habitudes de travail de la plus grande partie des bailleurs de fonds, l'autre lié à la conjoncture. Les actions humanitaires comme l'aide au développement se sont longtemps caractérisées par un refus systématique de tout processus d'évaluation externe. Et, les rares fois où ces évaluations eurent lieu, elles consistèrent en des vérifications comptables des plus sommaires. Que les pièces comptables soient parfaitement en ordre ne signifie aucunement que les produits achetés ont bel et bien été livrés à leurs destinataires ! De plus, les rares déplacements sur le terrain s'apparentèrent plus à ces 
visites guidées pour « camarades étrangers » dont les ex-pays socialistes se firent une spécialité. Enfin, certains échos des célébrations du cinquième centenaire de la Découverte (localement, « de la Conquête ») ne contribuèrent pas peu à accentuer les dérives prévaricatrices. On se souvient comment ces célébrations furent l'occasion du surgissement d'un double discours pour le moins contradictoire : une discussion assortie d'une remise à jour, tout à fait bien venue par ailleurs, des thèses lascasiennes ; une vulgate sur la dignité bafouée et les nécessaires réparations sonnantes et trébuchantes des monstruosités du colonialisme. Largement relayées par l'appareil sandiniste et certains démagogues miskitu comme Steadman Fagoth, ces vulgates sur le praetium doloris devinrent des sortes de lieux communs. Du coup se fit jour l'idée, tant chez certains donateurs que chez certains récipiendaires, que les projets de développement étaient autant de rentes. Et l'idée que ces dons étaient destinés à pallier des manques et à permettre de financer ce que l'on commençait déjà à appeler un développement durable et autosuffisant apparut comme largement désuète.

\section{Mitch : désastre naturel, aide d'urgence et nouvelle expérience de la catastrophe}

Bien que l'ouragan Mitch n'ait pas directement affecté la Côte atlantique nicaraguayenne, le bassin versant du Río Coco connut des crues et des inondations d'ampleur tout à fait exceptionnelle. Ainsi les eaux du Wangki et de ses affluents montèrent de telle sorte que le plus gros des cultures vivrières - riz, haricot, maïs, banane et autres musacées, manioc - , ainsi que de nombreux arbres fruitiers furent emportés. De même, toute une partie du cheptel - bovin, équin et porcin - comme des animaux de basse-cour périt noyée. Une bonne moitié des villages riverains des fleuves furent détruits en totalité. De plus, dans beaucoup de villages, où l'habitat ne fut que peu endommagé, la montée des eaux entrâna néanmoins la contamination de l'ensemble des puits et priva les habitants d'eau potable. Si la présence d'esprit des habitants menacés par la montée des eaux permit leur évacuation à temps, leur survie n'en fut pas moins particulièrement diffïile. La plupart d'entre eux survécut en mangeant des bananes plantains récoltées dans des plantations situées dans les collines ainsi que des produits de la chasse et de la pêche. Se procurer de l'eau potable devint une opération pour le moins problématique. Les villageois dont l'habitat avait été détruit se réinstallèrent sur les collines dans des huttes de branchages et de palmes à l'image de celles qu'ils construisent auprès des plantations éloignées des villages. Les mêmes n'eurent généralement plus d'autres vêtements que ceux qu'ils portaient lors de la montée des eaux, n'ayant en outre à leur disposition que très peu d'instruments aratoires et de cuisine et, le plus souvent, aucun médicament. Une machette, une marmite, une bassine ou un seau constituaient la seule et unique richesse de ces familles. Les déplacements sur le fleuve devinrent très diffíciles car bateaux et pirogues, coulés ou emportés, avaient fait les frais des crues. C'est dire que toute une population d'agriculteurs sédentaires se vit transformée du jour au lendemain en collecteurschasseurs et que la moitié d'entre elle devint de surcroît nomade.

Cette expérience de la détresse matérielle due à un cataclysme naturel s'accompagna de la sensation d'expérimenter une nouvelle catastrophe semblable à celle de la destruction des villages à l'époque de la guerre. Pis, cette sensation se conjugua à un sentiment d'abandon complet de la part des autorités nicaraguayennes. L'aide de 
première urgence apportée aux sinistrés fut ainsi acheminée de façon à la fois relativement tardive et chaotique. En amont de Raïti elle fut distribuée par l'armée nicaraguayenne et la défense civile largement débordées l'une et l'autre par l'ampleur de la tâche. En aval, une aide en vêtements et en nourriture fut collectée localement dans toute la RAAN par les Églises morave et catholique et convoyée par des commerçants locaux jusqu'à Waspam. De même, les agriculteurs de Francia Sirpi, Santa Clara et Awastingni - la zone des Llanos entre Puerto Cabezas et Waspam - envoyèrent des sacs de haricot et de maïs sur le fleuve. Mais, faute de bateaux, ceux-ci furent fort mal distribués. Par ailleurs, des membres de l'infanterie de marine des Pays-Bas et des marins de la flotte britannique portèrent assistance deux semaines durant aux populations du haut cours du Río Wangki. Une quarantaine de Hollandais bientôt relayés par des Britanniques aidèrent à déblayer le village de Raïti et à y construire des latrines. Un hélicoptère de la Royal Navy fut mis à disposition de l'Église morave afin d'effectuer une inspection des différentes communautés sinistrées. D'autres appareils furent affectés au transport de la nourriture et des médicaments acheminés par le PAM et le CNE, et à l'évacuation des blessés soignés sur les navires porte-hélicoptères. Enfin, le ministère de la santé finit par dépêcher dans la région dix-sept brigades de santé fortes de 70 médecins et infirmiers. Il convient de souligner combien les habitants eurent l'impression d'être non seulement livrés à eux-mêmes mais, plus encore, d'être considérés comme des citoyens de seconde zone. En effet, alors qu'ils avaient, à l'instar d'autres sinistrés, tout perdu et qu'ils se rendaient compte en écoutant la radio de la similitude de leur sort avec celui des sinistrés du versant pacifique du pays, aucun membre du gouvernement, aucun représentant des médias nationaux ou internationaux ne vint s'enquérir de leur sort. De même furent-ils particulièrement choqués par le refus de nombreux membres des brigades de santé de venir leur porter secours in situ.

Ce sentiment d'abandon fut redoublé par les ratés de la distribution de l'aide internationale dans les semaines qui suivirent l'ouragan. On se souviendra que dans le nord-ouest du pays, les démêlés entre les populations, les secouristes et le gouvernement donnèrent lieu à des débats publics largement relayés par la presse nationale et internationale. La dénonciation des détournements de l'aide par le gouvernement eut certainement pour effet de limiter les prévarications. Elle permit aussi aux sinistrés de sentir qu'ils pouvaient être entendus, ce qui facilita de toute évidence tout un travail de deuil. Â l'inverse, sur le fleuve, les choses fonctionnèrent en une manière de huis clos, où les victimes de la catastrophe furent chaque fois mises devant le fait accompli sans que leurs demandes ou leurs protestations ne soient jamais entendues et relayées. Le premier acte de ce huis clos tint à la rumeur selon laquelle certains des membres des brigades de santé détournèrent des médicaments jugés " trop bons » pour les habitants du fleuve. Le second prit la forme d'un détournement pur et simple d'une partie de la nourriture destinée aux sinistrés. Si une agence comme la Croix rouge nordaméricaine eut à cœur de surveiller l'acheminement des vivres, d'autres n'eurent pas cette sagesse. Certains des convoyeurs du PAM revendirent ainsi en toute impunité des cargaisons de nourriture à des commerçants honduriens et nicaraguayens. Parallèlement les employés de la mairie de Waspam, chapeautés par le maire libéral de la localité - Gerardo Tomas - , pillèrent allégrement les stocks de nourriture et de vêtements destinés aux sinistrés. Le troisième tint à l'incapacité de certaines agences 
internationales à s'adapter aux spécificités des demandes et des besoins des populations locales. Ainsi, dans son désir de distribuer rapidement des tôles aux habitants du haut cours du Río, la Croix rouge belge ne se préoccupa nullement de savoir comment celles-ci seraient transportées. Convoqués pour recevoir leur dû dans un bourg - Wivili, très en amont de leurs communautés - et dépourvus de tout moyen de transport, les bénéficiaires n'eurent d'autre solution que de revendre au plus offrant. Plus grave, on ne sut pas assez écouter les conseils de certaines oNG locales, telles Pana-pana ou FURCA. Celles-ci soulignèrent presque immédiatement que le plus important était sans doute moins d'acheminer de la nourriture que des semis, des instruments aratoires, des bateaux afin que les comunitarios retrouvent au plus vite l'autosuffisance alimentaire qui était naguère la leur. En effet, du fait de la crue et des dépôts de limons, beaucoup de parcelles étaient extrêmement fertiles. Au lieu de cela, on écouta les politiciens locaux trop heureux d'avoir à la fois des biens à détourner et d'apparaître comme les pourvoyeurs de leurs électeurs. Enfin, alors que le forage de puits, permettant d'avoir à nouveau accès à l'eau potable, était une autre des priorités, aucune des agences internationales n'y vit une mesure d'urgence pour laquelle devaient être mobilisés du matériel de forage et des moyens pour le transporter. De même, prit-on au départ comme critère pour déclarer une communauté sinistrée le degré de destruction de l'habitat mais d'aucune façon l'état des puits ou des sources, du cheptel et du domaine agricole, alors que, de fait, la disparition des points d'approvisionnement en eau potable, la perte des futures récoltes ou des arbres fruitiers était à moyen terme beaucoup plus dommageable que celle de l'habitat.

Ainsi, pour les habitants des communautés riveraines du Río Coco, l'ouragan Mitch n'eut pas pour seule conséquence des destructions matérielles. La brutalité et l'imprévisibilité des destructions causées par la tempête, les dysfonctionnements de l'aide aux sinistrés tout comme certaines bévues des ONG ou des agences internationales accréditèrent chez beaucoup des sentiments propres à grever les tentatives d'aides au développement dans la région. Le premier prit la forme d'une perte de foi dans l'avenir et dans les tentatives de développement endogènes : " $\mathrm{A}$ quoi bon travailler, si le fleuve peut emporter en quelques heures le travail d'une dizaine d'années ». Le second se traduisit par une valorisation des actions prosaïques les plus immédiates et souvent même par une valorisation des conduites délinquantes : «Pourquoi ne pas piller l'aide internationale, pourquoi ne pas la revendre si d'autres le font sans être punis ». Le troisième fut de croire que l'aide constituait une sorte de manne inépuisable et serait versée de manière ininterrompue.

\section{La manne de la reconstruction post-Mitch et ses effets pervers}

Une fois la première urgence passée, les habitants du Río Coco se trouvèrent confrontés à l'arrivée d'une aide sans précédent. En effet, eu égard à l'importance des sommes débloquées par la communauté internationale, tant pour la reconstruction du pays que pour lutter contre la pauvreté, bon nombre d'oNG et d'agences internationales jusqu'alors peu ou pas présentes dans la région firent leur apparition et entrèrent en concurrence avec les ONG déjà implantées sur place. Certaines agences internationales, telle la mission nicaraguayenne de l'OEA, vinrent concurrencer certaines ONG nicaraguayennes qui, de fait, avaient un quasi-monopole sur certaines régions, comme 
le haut cours du Río Coco et du Río Bocay. Plus en aval, l'ue relança un projet avec des moyens infiniment plus importants que ceux des ONG nicaraguayennes. L'arrivée de ces nouveaux intervenants s'inscrivit aussi de toute évidence dans des visées politiques. Le gouvernement nicaraguayen d'Arnoldo Alemán entendit contrer le poids grandissant de certaines ONG sandinistes, comme ALISTAR, dans le Bocay. De son côté, le gouvernement suédois qui finança le projet Hijos del río, exécuté par l'OEA, ne souhaita pas financer des ONG par trop ouvertement affiliées à un parti politique. Plus acquis aux sandinistes qu'aux anciens contras ou au gouvernement, certains fonctionnaires de l'ue ne furent pas mécontents de constituer un pôle indépendant de l'administration libérale dans la RAAN et le municipe de Waspam. Cette concurrence politique fut aussi le fait des ONG et des institutions locales elles-mêmes. La mairie de Waspam s'opposa ainsi férocement à certaines initiatives d'ONG comme FADCANIC ou Wangki Lumpia, toutes deux entre les mains de responsables sandinistes locaux. Les uns et les autres avaient en vue les prochaines élections municipales (2001) et entendaient rentabiliser politiquement les retombées de l'aide. Enfin, comme on l'a vu, des conceptions différentes de l'aide au développement furent en concurrence.

Cette situation d'abondance de moyens ne va pas sans effets pervers, d'autant que l'aide devait en principe être dépensée selon un calendrier fixé d'avance qui tint plus compte des impératifs de la comptabilité des pays donateurs que de certains aléas locaux. Beaucoup d'oNG se chargèrent du plus grand nombre de projets possibles sans toujours être en mesure de les réaliser. De plus, ces fonds furent trop souvent attribués sur la bonne mine de leurs dirigeants, ou de leur site Internet, et beaucoup plus rarement suite à des visites in situ et des discussions avec les sinistrés. Certaines ONG firent monter les enchères de la manière la plus incroyable. Quelques-unes n'hésitèrent pas à promettre des commissions ou des gratifications à des personnalités locales - responsables d'associations locales, maire et membres des conseils municipaux, gouverneur et conseillers régionaux —, dans le dessein d'être désignées pour exécuter telle ou telle opération. Elles purent aussi pratiquer la " guerre des tarifs » contre une organisation rivale. Ainsi dans le haut Río Coco, ALISTAR tentera à plusieurs reprises de monter les responsables d'associations locales contre l'ora en affirmant que celle-ci devrait mieux rémunérer les habitants participant aux projets de reconstruction ou distribuer plus de nourriture. Et, dans le municipe de Waspam, c'est plus d'une fois que FURCA et Pana-pana s'entendirent dire que d'autres ONG n'exigeaient aucun travail de la part des bénéficiaires des projets de développement. De même se virent-elles reprocher de pratiquer des prêts sans intérêts plutôt que de distribuer des biens gratuitement. Ces multiples marchandages n'améliorèrent nullement le sort des sinistrés; ils se traduisirent avant tout par de considérables retards dans l'exécution de certains projets. De telles pratiques ne furent d'ailleurs pas dépourvues d'inconvénients pour leurs principaux parrains : pour n'en citer qu'un exemple, ALISTAR ne tarda pas à voir certaines communautés ou associations indiennes locales se mettre à pratiquer à son encontre le chantage qu'elle-même leur avait conseillé de mettre en place avec l'OEA.

L'abondance des ressources favorisa une dépendance croissante vis-à-vis de produits mal adaptés à l'environnement et aux conditions écologiques, tel le parpaing de béton ou la tôle galvanisée. L'idéal devint en effet désormais la maison en parpaing avec un toit de tôle, habitat dont on sait pourtant qu'il se transforme durant six mois 
de l'année en fournaise parfaitement inhabitable. Mais, n'est-il pas plus facile pour une ONG de distribuer des tôles ou du ciment, plutôt que de faire venir des charpentiers et des maçons capables d'enseigner aux habitants à couper le bois ou le bambou et à le traiter, ou à produire des tuiles et des briques ? De telles opérations nécessitent que l'on soit au contact de la population, communauté par communauté, et que l'on soit capable de s'installer durablement dans des endroits parfois très inhospitaliers pour y vivre et y travailler en deçà de toute visibilité médiatique. Du coup, grâce à certains démagogues pressés de faire valoir des résultats immédiats, l'idée que l'on puisse améliorer la fabrication des toits en palmes et en bardeau ou la confection des tuiles fut perçue comme un refus raciste de faire bénéficier les Indiens du progrès. Les propositions de constructions en bois, en bambou ou en adobe furent aussi souvent interprétées de la même façon. Et dans les zones du Bocay et du haut Río Coco, les sinistrés ne commencèrent à reconsidérer le problème qu'après s'être aperçus que l'OEA avait opté pour ses propres bâtiments de service et de logement en faveur de la tuile et/ou de la palme.

Le dernier effet pervers de l'aide tint à sa politisation au plus mauvais sens du terme. L'aide à la reconstruction et les fonds pour la lutte contre la grande pauvreté furent pensés comme s'inscrivant dans un projet global impliquant l'ensemble des communautés et ses membres les plus déshérités. Or, de fait, certaines maladresses donnèrent nombre d'arguments à ceux qui affirmaient que les oNG étaient autant d'acteurs venant appuyer telle ou telle force politique et non promouvoir le développement. On a vu que certaines agences internationales apparurent d'emblée comme constituant des sortes de contre-poids aux équilibres politiques locaux. Si l'OEA et les donateurs suédois du projet Hijos del río se gardèrent très soigneusement d'entrer en politique, les responsables du projet de l'uE n'eurent pas ces pudeurs. Les premiers mirent un point d'honneur à répartir équitablement les microcrédits sans jamais privilégier les familles d'affiliation libérale. Et ils firent de même chaque fois qu'ils suscitèrent la formation de comités ad hoc, que leurs interlocuteurs fussent ou non sandinistes. Hasard ou volonté délibérée, les seconds — les intervenants de l'UE, en l'occurrence - se prirent de fait à privilégier les secteurs affiliés au sandinisme. Et parfois même de la façon la plus grossière qui soit comme lorsqu'ils offrirent des facilités de transport aux activistes du front durant la campagne électorale des municipales. Ce faisant, ils contribuèrent à accréditer l'idée que l'activité politique n'est en rien la poursuite et la réalisation d'intérêts collectifs, idéal déjà passablement malmené dans la région, mais bien et avant tout un moyen d'obtenir des gratifications individuelles.

\section{Les ratés de l'aide}

Par-delà ces effets pervers, il convient de noter que l'aide connut aussi bon nombre de ratés. Certains portent à sourire et relèvent d'un scénario à la Courteline. Ainsi, ces latrines aux parois de zinc données par la Croix rouge nord-américaine, qui offrent l'avantage de se transformer en four solaire dès que le soleil brille, mais qui, de ce fait, sont tout simplement inutilisables une bonne partie de l'année. On pourrait citer aussi le cas de ce logisticien de MSF s'obstinant à vouloir qu'un motoriste novice en matière de rapide franchisse les premiers étroits en aval de Raïti, pourtant réputés dangereux : 
la pirogue se retourna et un matériel de forage relativement coûteux sombra dans un trou d'eau. Beaucoup l'avait pourtant prévenu de la nécessité de faire appel pour cette partie du trajet à un pilote très expérimenté. Il y eut aussi les projets d'élevage porcin où l'on s'ingénia à importer à grands frais des animaux du versant pacifique. Parfaitement inadaptés au climat, ils ne tardèrent pas à mourir ; seuls quelques propriétaires avisés les mangèrent sans attendre l'épidémie. Pourtant, là encore, les conseils des gens du lieu n'avaient pas manqué. Tous soulignaient la nécessité d'agir comme l'avait fait vSF quelques années plus tôt : trouver des animaux adaptables au lieu, commencer avec un petit nombre et les laisser se reproduire. D'autres remarquaient que les porcs étaient facilement volés et que les gens en faisaient peu de cas. Ils ajoutaient en outre que le bétail bovin avait toujours été l'objet de plus d'attention, d'autant que les gens y voyaient là une sorte de tirelire pour le futur. Certains projets de répartition des silos furent pareillement menés en dépit du bon sens. On distribua sans chercher à savoir si les récipiendaires des silos seraient capables d'en prendre soin. Et, de fait, un nombre non négligeable d'entre eux furent revendus à des commerçants ou abandonnés à la rouille. Une politique de microcrédits, dans le style de celles qui sont pratiquées par Pana-pana ou FURCA auraient permis de discerner quels étaient les individus susceptibles de s'impliquer dans ces projets.

Au-delà, il est des échecs infiniment plus dommageables à moyen et à long termes. Nombre de projets de reconstruction de l'habitat ou de distribution de vivres, de vêtements et de médicaments ne furent pas menés à bien du fait du détournement des fonds qui leur étaient destinés. Le cas le plus exemplaire fut celui des ponts qui devaient être reconstruits sous la direction du Projet Waspam de la CE. Les constructeurs eurent tout le loisir de détourner et de revendre le ciment et les fers à béton destinés à ces constructions. Il en fut de même de certains projets de cadastrage des terres revendiquées par les communautés indigènes. Le maire de Waspam, Gerardo Tomas, et plusieurs de ses proches volèrent et revendirent les produits destinés aux sinistrés. L'édile put ainsi, non seulement mener grand train avec ses acolytes, mais acheter une propriété foncière aux dimensions tout à fait respectables et se lancer dans l'élevage bovin avec cent cinquante têtes de bétail. Une ONG liée au FSLN comme FADCANIC laissa à moitié achevées un nombre considérable de constructions abandonnées à l'état de squelettes dans différentes communautés. Il en est d'autres où rien ne fut fait. Enfin, l'enquête réalisée à grands frais par l'Université morave auprès des communautés indigènes sur l'avant-projet de loi concernant le régime des propriétés communales fut dans une large mesure une escroquerie. Les questionnaires destinés aux habitants des communautés riveraines du fleuve furent remplis sans que les enquêteurs daignent se rendre sur place (mais sans renoncer pour autant aux confortables frais de mission versés à cet effet).

Nul doute que de telles malversations tiennent à certaines habitudes de piratage fort anciennes dans la région; reste que certains des donateurs les ont, à bien des égards, favorisées. Rares sont ceux qui eurent la sagesse des Canadiens, des Danois, de la Fondation de France, des Hollandais ou des Suédois. Ceux-là réalisèrent de véritables inspections sur le terrain. Le personnel diplomatique suédois s'est rendu à plusieurs reprises dans les marges du département de Jinotega pour voir où en étaient les réalisations de Hijos del Río. FurCA a dû rendre des comptes à des experts indépendants recrutés par ses propres donateurs. On n'a rien vu de tel pour les projets 
d'Alistar, FADCANIC ou de l'Ue. Aucun fonctionnaire européen, aucun membre de l'AID, aucun personnel des différentes ambassades des pays donateurs ne jugea bon de se promener une semaine durant sur le fleuve, de s'arrêter dans les villages, d'y dormir, de parler avec les gens et de les écouter. Il est évident que de tels voyages ne sont pas la panacée universelle ; ils ont néanmoins certaines vertus et auraient sans doute permis de s'apercevoir assez tôt de différentes malversations. Ils auraient permis à tout le moins d'identifier les dysfonctionnements, de recevoir et d'appuyer les demandes en justice des victimes de ces escroqueries et, éventuellement, de suspendre quand il en était encore temps les versements destinés aux projets notoirement ineflicaces ou propices aux détournements de fonds. Le laxisme qui a prévalu chez beaucoup de donateurs n'a pas peu contribué à légitimer les pratiques les plus malhonnêtes et les discours schizophrènes. Pour beaucoup, les projets de reconstruction ou de lutte contre la pauvreté ne sont plus simplement articulés à des systèmes de clientélisme politique, ils sont devenus autant de possibilités de s'enrichir en toute impunité et encouragent de fait un conformisme délinquant aux visées les plus immédiates.

\section{Pour un changement des attitudes et des méthodes}

Le bilan en demi-teinte que l'on peut tracer des opérations de reconstruction et de développement dans les communautés du Wangki et de ses aflluents ne saurait conduire à prôner un quelconque retrait des donateurs internationaux. Il convient au contraire de réorienter cette aide en fonction de trois objectifs principaux, objectifs susceptibles de recueillir l'assentiment des populations locales, comme du gouvernement du Président Bolaños, effectivement engagé dans une lutte contre la corruption.

Il est un volant de l'aide internationale qui doit être affecté à des institutions, type OEA, seules capables de prendre en charge la logistique extrêmement complexe que supposent certaines opérations particulièrement lourdes, de déminage par exemple : les sites sont depuis longtemps identifiés, reste à mettre en place une opération de longue haleine. De même conviendrait-il de pouvoir appuyer une enquête comblant les lacunes des différentes études sur les problèmes fonciers. Beaucoup des projets de développement achoppent sur le fait que, pour obtenir des crédits permettant de lancer des cultures à forte valeur ajoutée ou des projets d'élevage d'animaux exotiques (cuirs de sauriens), les intéressés doivent pouvoir présenter des titres de propriété. Or, jusqu'à ce jour, les Miskitus continuent de vivre dans un régime foncier parfaitement indéfini. Certes, certains arrêts internationaux, comme dans le cas du différend entre la communauté d'Awastingni et l'État nicaraguayen, reconnaissent l'existence de terres indigènes que l'État n'est pas en droit de concéder a des entreprises étrangères. Mais cela ne constitue pas pour autant des titres de propriété pour les communautés : un cadastrage généralisé des terres indiennes serait un préalable aux projets de développement, et il conviendrait de rouvrir au sein des forum internationaux la question des droits d'usage de certaines communautés miskitus sur des terres honduriennes. Plusieurs de ces terres ont en effet été accaparées par des commerçants spéculateurs, et si d'autres y échappent encore, c'est uniquement parce qu'elles sont éloignées et dépourvues de voies d'accès terrestre.

Par ailleurs, il est avéré que les politiques de microcrédits ont dans l'ensemble été un succès, même si elles ne sont en rien spectaculaires - ceci expliquant peut-être 
cela. Elles supposent en effet des réseaux locaux présents sur le terrain de façon durable, c'est-à-dire susceptibles de toucher les communautés les plus reculées pour y proposer des prêts de 50,100,150 ou 200 dollars. De telles opérations ne peuvent être réalisées que par des opérateurs locaux qui ont grosso modo la même connaissance fine du terrain que les commerçants locaux qui pratiquent ce genre de prêts à des taux généralement usuraires. De tels prêts ont ceci de pertinent qu'ils répondent à des attentes précises des habitants qui empruntent d'ailleurs de la façon la plus sagace.

Il est aussi un style de conduite dont il conviendrait de se défaire, consistant à apporter un soin maniaque dans la surveillance et la conception des ouvrages destinés à abriter les administrateurs de projet, tandis que la plus grande négligence accompagne la construction d'équipements collectifs, conduite illustrée jusqu'à la caricature par la mise en cuvre du Projet Waspam.

Enfin, tous les projets d'aide au développement devraient prendre l'habitude de provisionner des fonds à la fois pour de réelles inspections sur le terrain mais aussi pour d'éventuelles actions en justice. Mieux, de telles décisions devraient être très largement discutées et annoncées lors des visites préalables à la mise en place des opérations de développement. Tout un programme!

Mexico, mai 2002

\author{
Gilles BATAILLON \\ CEMCA-CIDE (Mexico) \\ Centre d'étude des mouvements sociaux - EHESS (Paris)
}

NOTES

1. Cet article fait suite au texte publié dans la dernière Chronique (Journal de la Société des Américanistes, tome 87 [2001], pp. 376-392) où le lecteur trouvera une chronologie des événements de l'histoire récente du Nicaragua, ainsi qu'une liste des sigles correspondant aux principaux acteurs institutionnels et une bibliographie. Les matériaux nécessaires à cette étude ont été recueillis au cours de différentes missions en Moskitia nicaraguayenne, dont une (en août-septembre 2001) a été financée par la Fondation de France. Je suis bien entendu seul responsable des opinions et conclusions exprimées dans cet article.

2. La valeur du lempira oscillait, à l'époque, entre 25 et 50 centimes d'euro. 